\title{
VANISHING MOMENTS FOR SCALING VECTORS
}

\author{
DAVID K. RUCH
}

Received 20 August 2003

\begin{abstract}
One advantage of scaling vectors over a single scaling function is the compatibility of symmetry and orthogonality. This paper investigates the relationship between symmetry, vanishing moments, orthogonality, and support length for a scaling vector $\Phi$. Some general results on scaling vectors and vanishing moments are developed, as well as some necessary conditions for the symbol entries of a scaling vector with both symmetry and orthogonality. If orthogonal scaling vector $\Phi$ has some kind of symmetry and a given number of vanishing moments, we can characterize the type of symmetry for $\Phi$, give some information about the form of the symbol $P(z)$, and place some bounds on the support of each $\phi_{i}$. We then construct an $L^{2}(\mathbb{R})$ orthogonal, symmetric scaling vector with one vanishing moment having minimal support.
\end{abstract}

2000 Mathematics Subject Classification: 42-xx, 42C40, 42C15.

1. Introduction. In this paper, we will discuss conditions for vanishing moments for scaling vector $\Phi=\left(\phi_{1}, \ldots, \phi_{r}\right)^{T}$ solutions to the matrix refinement equation (MRE)

$$
\Phi(x)=\sum_{k=0}^{N} C_{k} \Phi(2 x-k)
$$

with $r \times r$ matrix coefficients $C_{k}$. Taking the Fourier transform of this MRE gives

$$
\hat{\Phi}(w)=P\left(\frac{w}{2}\right) \hat{\Phi}\left(\frac{w}{2}\right),
$$

where the 2-scale symbol $P$ is the $r \times r$ matrix

$$
P(w)=\frac{1}{2} \sum_{k=0}^{N} C_{k} e^{-i k w} \quad \text { or } \quad P(z)=\frac{1}{2} \sum_{k=0}^{N} C_{k} z^{k} \quad \text { with } z=e^{-i w} .
$$

We will use $p_{i j}(z)$ to denote the $i-j$ entry of $P$, and we will occasionally interchange $z$ and $w$ for the argument of the symbol $P$; the context should prevent confusion.

Scaling vectors and solutions to MREs have been studied in [1, 3, 5, 6, 7] and many others. In particular, Heil and Colella [5] showed that the eigenvalues of $\Delta^{\infty}=\lim _{j \rightarrow \infty} P^{j}(1)$ are crucial to understanding solutions of the MRE. The unconstrained solutions are precisely those which are the compactly supported distributional solutions to the MRE (1.1). For this reason, we will restrict our attention in this paper to unconstrained solutions to (1.1). Indeed, all fundamental solutions of scalar refinement equations are of this type. The vast majority of scaling vector examples in the literature are unconstrained. 
DEFINITION 1.1. Solutions to the MRE (1.1) are defined to be unconstrained if $\Delta^{\infty}$ exists and is nontrivial: $P(0)$ has 1 for a nondegenerate eigenvalue, and all other eigenvalues have modulus less than 1 .

It is also shown in [5] that unconstrained MRE solutions $\Phi$ satisfy

$$
\hat{\Phi}(1)=\left(\int_{\mathbb{R}} \phi_{1}, \ldots, \int_{\mathbb{R}} \phi_{r}\right)^{T} \neq \mathbf{0} .
$$

As is standard in the literature, we say the polynomial accuracy $p$ of a scaling vector is the maximum integer $p$ for which $x^{k} \in V_{0}$ for $k=0,1, \ldots, p-1$. A common sufficient condition for the density condition of a multiresolution analysis (MRA) generated by $\Phi$ requires polynomial accuracy at least $p \geq 1$ (e.g., [3]). Throughout this paper, all scaling vectors are assumed to have accuracy $p \geq 1$.

The following combines some important results on accuracy and smoothness found in $[1,7]$ necessary for our discussion.

THEOREM 1.2. Suppose that compactly supported scaling vector $\Phi$ generates an MRA. The following are equivalent:

(1) $\Phi$ provides polynomial accuracy $m$;

(2) the elements of $P(w)$ are trigonometric polynomials and there are vectors $\mathbf{y}^{k}$ for $k=0, \ldots, m-1$ satisfying

$$
\begin{gathered}
\sum_{k=0}^{m}\left(\begin{array}{c}
m \\
k
\end{array}\right)\left(\mathbf{y}^{k}\right)^{T}(2 i)^{k-m}\left(D^{m-k} P\right)(0)=2^{-m}\left(\mathbf{y}^{m}\right)^{T}, \\
\sum_{k=0}^{m}\left(\begin{array}{c}
m \\
k
\end{array}\right)\left(\mathbf{y}^{k}\right)^{T}(2 i)^{k-m}\left(D^{m-k} P\right)(\pi)=\mathbf{0}^{T} ;
\end{gathered}
$$

(3) the symbol $P(z)$ factors as

$$
P(z)=\frac{1}{2^{m}} C_{x_{0}}\left(z^{2}\right) \cdots C_{x_{m-1}}\left(z^{2}\right) P_{m}(z) C_{x_{m-1}}(z)^{-1} \cdots C_{x_{0}}(z)^{-1} .
$$

In this case, if inf $\gamma_{k}<m-1 / 2$, then $\Phi \in L^{2}(\mathbb{R})$, where

$$
\gamma_{k}=\frac{1}{k} \log _{2} \sup _{\omega}\left\|P_{m}\left(\frac{\omega}{2}\right) \cdots P_{m}\left(\frac{\omega}{2^{k}}\right)\right\| .
$$

Whether the MRA generated by $\Phi$ is orthogonal is determined by the following condition found in [10].

THEOREM 1.3. The scaling vector $\Phi$ generates an orthogonal basis if and only if its symbol P satisfies

$$
P(z) P^{*}\left(\frac{1}{z}\right)+P(-z) P^{*}\left(\frac{-1}{z}\right)=I_{r}
$$

One advantage to scaling vectors over a single scaling function is the compatibility of symmetry and orthogonality, as seen in [3] and elsewhere. The symmetry condition given below for a scaling vector was proved by Plonka and Strela and appears in [7]. 
THEOREM 1.4. A scaling vector $\Phi$ has component functions that are symmetric or antisymmetric if and only if there exists a matrix $E(z)=\operatorname{diag}\left( \pm z^{2 T_{1}}, \ldots, \pm z^{2 T_{r}}\right)$ for which

$$
P(z)=E\left(z^{2}\right) P\left(z^{-1}\right) E^{-1}(z),
$$

where the $T_{j}$ are the points of symmetry for the $\phi_{j}$ and the \pm sign is + when $\phi_{j}$ is symmetric and - when antisymmetric.

When $r=2$ and $T_{1}=0$, the condition $P(z)=E\left(z^{2}\right) P\left(z^{-1}\right) E^{-1}(z)$ is equivalent to the following, useful in the sequel:

$$
\begin{aligned}
& p_{11}(z)=p_{11}\left(z^{-1}\right), \quad p_{12}(z)= \pm z^{-2 T_{2}} p_{12}\left(z^{-1}\right), \\
& p_{21}(z)= \pm z^{4 T_{2}} p_{21}\left(z^{-1}\right), \quad p_{22}(z)=z^{2 T_{2}} p_{22}\left(z^{-1}\right) .
\end{aligned}
$$

REMARK 1.5. We will say that a scaling vector $\Phi$ is symmetric if all component functions of $\Phi$ are either symmetric or antisymmetric.

The following result, needed in the sequel, shows that the length of each polynomial entry in the symbol is unaffected by integer shifts of the scaling functions.

Proposition 1.6. Let $\Phi=\left(\phi_{1}, \phi_{2}\right)^{T}$ be a scaling vector with symbol P. If each $\phi_{k}$ is shifted by $S_{k} \in \mathbb{Z}$, the symbol of the new scaling vector $\Phi_{*}$ is

$$
P_{*}(z)=\left(\begin{array}{cc}
z^{-S_{1}} p_{11}(z) & z^{S_{2}-2 S_{1}} p_{12}(z) \\
z^{S_{1}-2 S_{2}} p_{21}(z) & z^{-S_{2}} p_{22}(z)
\end{array}\right)
$$

Proof. Note that $\hat{\Phi}_{*}(z)=T(z) \hat{\Phi}(z)$, where $T(z)=\operatorname{diag}\left\{z^{-S_{1}}, z^{-S_{2}}\right\}$. Substituting into (1.2), we have

$$
\begin{gathered}
T^{-1}\left(z^{2}\right) \hat{\Phi}_{*}\left(z^{2}\right)=P(z) T^{-1}(z) \hat{\Phi}_{*}(z), \\
\hat{\Phi}_{*}\left(z^{2}\right)=T\left(z^{2}\right) P(z) T^{-1}(z) \hat{\Phi}_{*}(z) \\
=\left(\begin{array}{cc}
z^{-S_{1}} p_{11}(z) & z^{S_{2}-2 S_{1}} p_{12}(z) \\
z^{S_{1}-2 S_{2}} p_{21}(z) & z^{-S_{2}} p_{22}(z)
\end{array}\right) \hat{\Phi}_{*}(z) .
\end{gathered}
$$

2. Scaling vectors with vanishing moments. The study of vanishing moments for a single scaling function is discussed in [2] and many other papers. In [2], Daubechies shows how to link the symbol's condition for vanishing moments with the condition for polynomial accuracy via Bezout's theorem to obtain a family of coiflets. In the scaling vector situation, the symbol is a matrix, and matters are more complex. In this section, we develop some general results on scaling vectors and vanishing moments, as well as some necessary conditions for the symbol entries of a scaling vector with both symmetry and orthogonality. We first characterize the vanishing moments of $\Phi$ in terms of the derivatives of its symbol $D^{k} P(0)$ according to the following result, first presented in [8]. 
DEFINITION 2.1. $\Phi=\left(\phi_{1}, \ldots, \phi_{r}\right)^{T}$ has $L-1$ vanishing moments if

$$
\int x^{n} \phi_{i}(x) d x=0 \text { for } n=1, \ldots, L-1
$$

for all $\phi_{i}$.

THEOREM 2.2. The scaling vector $\Phi=\left(\phi_{1}, \ldots, \phi_{r}\right)^{T}$ generating an MRA has $L-1$ vanishing moments if and only if $\hat{\Phi}(0)$ is a 0 -eigenvector of $D^{k} P(0)$ for $k=1, \ldots, L-1$.

Proof. As noted in (1.4), $\hat{\Phi}(0) \neq \mathbf{0}$. Differentiating the MRE (1.2) $k$ times with respect to $\omega$ yields

$$
\hat{\Phi}^{(k)}(\omega)=\frac{1}{2^{k}} \sum_{i=0}^{k}\left(\begin{array}{l}
k \\
i
\end{array}\right) D^{k-i} P\left(\frac{\omega}{2}\right) \hat{\Phi}^{(i)}\left(\frac{\omega}{2}\right) .
$$

Suppose $\Phi$ has $L-1$ vanishing moments. Recall from Fourier analysis that $\Phi$ having $L-1$ vanishing moments is equivalent to

$$
\hat{\Phi}^{(i)}(0)=\mathbf{0}
$$

for $i=1, \ldots, L-1$. Now let $k \in\{1, \ldots, L-1\}$. From the theorem hypothesis, (2.2) at $\omega=0$ reduces to

$$
\mathbf{0}=\frac{1}{2^{k}} D^{k} P(0) \hat{\Phi}^{(0)}(0)
$$

so $\hat{\Phi}(0)$ is a 0-eigenvector of $D^{k} P(0)$.

Suppose $\hat{\Phi}(0)$ is a 0 -eigenvector. We use the principle of strong induction. Define $S=\left\{n \in \mathbb{N}\right.$ : if $\hat{\Phi}(0)$ is a 0 -eigenvector of $D^{k} P(0)$ for $k=1, \ldots, n$, then $\Phi$ has $n$ vanishing moments . To see that $1 \in S$, assume $\hat{\Phi}(0)$ is a 0 -eigenvector of $D P(0)$. From (2.2) at $\omega=0$ and $k=1$, we get

$$
\hat{\Phi}^{(1)}(0)=\frac{1}{2^{1}} D P(0) \hat{\Phi}^{(0)}(0)+P(0) \hat{\Phi}^{(1)}(0)=\frac{1}{2} P(0) \hat{\Phi}^{(1)}(0) .
$$

Thus $P(0) \hat{\Phi}^{(1)}(0)=2 \hat{\Phi}^{(1)}(0)$, so either $\hat{\Phi}^{(1)}(0)$ is a 2-eigenvector of $P(0)$ or $\hat{\Phi}^{(1)}(0)=\mathbf{0}$. However, since we have an MRA, $P(0)$ has spectral radius 1 by [6, Proposition 1.1], which forces $\hat{\Phi}^{(1)}(0)=\mathbf{0}$. Thus $1 \in S$.

Next assume that $\{1, \ldots, k\} \subset S$. To see that $k+1 \in S$, assume that $\hat{\Phi}(0)$ is a 0 eigenvector of $D^{i} P(0)$ for $i=1, \ldots, k+1$. Since $\{1, \ldots, k\} \subset S$, $\Phi$ has $k$ vanishing moments. From this and (2.2) at $\omega=0$ with $k+1$, we have

$$
\begin{aligned}
\hat{\Phi}^{(k+1)}(0) & =\frac{1}{2^{k+1}} \sum_{i=0}^{k+1}\left(\begin{array}{c}
k+1 \\
i
\end{array}\right) D^{k+1-i} P(0) \hat{\Phi}^{(i)}(0) \\
& =\frac{1}{2^{k+1}} P(0) \hat{\Phi}^{(k+1)}(0) .
\end{aligned}
$$

Thus $\hat{\Phi}^{(k+1)}(0)$ is a $2^{k+1}$-eigenvector of $P(0)$ or $\hat{\Phi}^{(k+1)}(0)=\mathbf{0}$. Since $P(0)$ has spectral radius $1, \hat{\Phi}^{(k+1)}(0)=\mathbf{0}$. Thus $k+1 \in S$. 
We now record some useful results about vanishing moments and symmetric scaling vectors.

Proposition 2.3. If $f(x)$ is symmetric about some $\beta \neq 0$, and $\int_{\mathbb{R}} x f(x) d x=0$, then $\int_{\mathbb{R}} f(x) d x=0$.

Proof. Define $g(u)=u f(u+\beta)$ and note that $g$ is odd. Thus $\int_{\mathbb{R}} g(u) d u=0$, so letting $x=u+\beta$, we have $\int_{\mathbb{R}} x f(x) d x=\beta \int_{\mathbb{R}} f(x) d x$ whence $\int_{\mathbb{R}} f(x) d x=0$.

COROLLARY 2.4. If $\Phi$ is a symmetric scaling vector with a vanishing moment, then at least one $\phi_{k}$ must be symmetric about 0 .

Proof. $\hat{\Phi}(0)$ cannot vanish as noted in (1.4). Apply Proposition 2.3.

THEOREM 2.5. Suppose $\Phi=\left(\phi_{1}, \ldots, \phi_{r}\right)^{T}$ generates a nontrivial orthonormal basis. Then not all $\phi_{i}$ can be symmetric about integers, and not all $\phi_{i}$ can be antisymmetric.

Proof. That not all $\phi_{i}$ can be antisymmetric follows immediately from Corollary 2.4 .

To prove that not all $\phi_{i}$ can be symmetric, we prove the contrapositive. Begin by shifting each $\phi_{i}$ so that it is symmetric about 0 , if necessary. Now let $P(z)$ be the symbol of this shifted $\Phi$. By the symmetry Theorem $1.4, P(z)=P(1 / z)$ for all $z$. Thus the $k$ - $k$ entry of $\theta=P(z) P^{*}(1 / z)+P(-z) P^{*}(-1 / z)$ is

$$
\theta_{k k}=\sum_{j=1}^{r} p_{k j}^{2}(z)+p_{k j}^{2}(-z) .
$$

Letting $n_{k j}$ denote the degree of the highest-degree nonzero term $a_{n_{k j}} z^{n_{k j}}$ of $p_{k j}(z)$ and $n_{k}=\max _{j}\left(n_{k j}\right)$, we see that $\theta_{k k}$ has $2 n_{k}$ degree term

$$
\sum_{n_{k j}=n_{k}}\left(a_{n_{k j}} z^{n_{k j}}\right)^{2}+\left(a_{n_{k j}}(-z)^{n_{k j}}\right)^{2}=2 z^{2 n_{k}} \sum_{n_{k j}=n_{k}}\left(a_{n_{k j}}\right)^{2} \neq 0 .
$$

Since the basis is nontrivial, $P(z)$ is not constant, so $n_{k}>0$ for at least one value of $k$ whence $\theta_{k k}$ is not constant for this $k$ value. Thus $\theta$ is not the identity matrix, and so by (1.8), $\Phi$ does not generate an orthonormal basis.

We introduce the notation $\operatorname{degL}(R)=n-m$ for a polynomial $R(z)=a_{m} z^{m}+\cdots+$ $a_{n} z^{n}$, where $m \leq n$. The following results are useful for analyzing the symbol $P(z)$ when scaling vector $\Phi$ is symmetric.

LEMMA 2.6. Let polynomial $R(z)=a_{m} z^{m}+\cdots+a_{n} z^{n}$ satisfy $z^{k} R(z)= \pm R\left(z^{-1}\right)$, where $a_{m}, a_{n} \neq 0, m \leq n$. Then $m=-n-k$ and $\operatorname{degL}(R)=2 n+k$ is even if and only if $k$ is even.

Proof. The condition $z^{k} R(z)= \pm R\left(z^{-1}\right)$ can be rewritten as follows:

$$
a_{m} z^{m+k}+\cdots+a_{n} z^{n+k}= \pm\left(a_{m} z^{-m}+\cdots+a_{n} z^{-n}\right) .
$$

Then the highest-degree terms of (2.9) must be $a_{n} z^{n+k}= \pm a_{m} z^{-m}$ so $n+k=-m$ whence $\operatorname{degL}(R)=n-m=2 n+k$. 
Proposition 2.7. Suppose symmetric $\Phi=\left(\phi_{1}, \phi_{2}\right)^{T}$ generates a nontrivial orthonormal basis with the $\phi_{k}$ (anti)symmetric about integers. Then

$$
\operatorname{degL}\left(p_{11}\right)=\operatorname{degL}\left(p_{12}\right), \quad \operatorname{degL}\left(p_{21}\right)=\operatorname{degL}\left(p_{22}\right)
$$

Proof. By Theorem 2.5, we may assume $\phi_{1}$ is symmetric and $\phi_{2}$ is antisymmetric. Then shift each $\phi_{k}$ to be (anti)symmetric about 0 . Note that this does not affect $\operatorname{degL}\left(p_{j k}\right)$ for any $j, k$ by Proposition 1.6. Letting $P(z)$ denote the symbol of this shifted scaling vector, Theorem 1.4 is satisfied with $E(w)=\operatorname{diag}\{1,-1\}$; so $p_{11}(z)=p_{11}\left(z^{-1}\right)$, $p_{12}(z)=-p_{12}\left(z^{-1}\right), p_{21}(z)=-p_{21}\left(z^{-1}\right), p_{22}(z)=p_{22}\left(z^{-1}\right)$, and so the $k-k$ entry of $\theta=P(z) P^{*}(1 / z)+P(-z) P^{*}(-1 / z)$ is

$$
\theta_{k k}=p_{k 1}^{2}(z)+p_{k 1}^{2}(-z)-\left(p_{k 2}^{2}(z)+p_{k 2}^{2}(-z)\right)
$$

Since $\theta_{k k}=1$ because of the orthogonality, $\operatorname{deg}\left(p_{k 1}^{2}(z)\right)=\operatorname{deg}\left(p_{k 2}^{2}(z)\right)$. Thus $\operatorname{degL}\left(p_{k 1}\right)=$ $\operatorname{degL}\left(p_{k 2}\right)$.

Proposition 2.8. Suppose symmetric $\Phi=\left(\phi_{1}, \phi_{2}\right)^{T}$ generates a nontrivial orthonormal basis with $\phi_{1}$ symmetric about an integer and $\phi_{2}$ symmetric about a half integer. Then

$$
\operatorname{degL}\left(p_{11}\right)+1 \leq \operatorname{degL}\left(p_{12}\right), \quad \operatorname{degL}\left(p_{21}\right)+1 \leq \operatorname{degL}\left(p_{22}\right)
$$

Proof. Shift $\phi_{1}$ and $\phi_{2}$ to be symmetric about 0 and $-1 / 2$, respectively. Note that this does not affect $\operatorname{degL}\left(p_{j k}\right)$ for any $j, k$ by Proposition 1.6. Letting $P(z)$ denote the symbol of this shifted scaling vector, Theorem 1.4 is satisfied; so by $(1.10), p_{11}(z)=$ $p_{11}\left(z^{-1}\right), z^{-1} p_{12}(z)=p_{12}\left(z^{-1}\right), p_{21}(z)=z^{-2} p_{21}\left(z^{-1}\right), p_{22}(z)=z^{-1} p_{22}\left(z^{-1}\right)$, and so the $1-1$ entry of $\theta=P(z) P^{*}(1 / z)+P(-z) P^{*}(-1 / z)$ is

$$
\theta_{11}=p_{11}^{2}(z)+p_{11}^{2}(-z)+z^{-1} p_{12}^{2}(z)-z^{-1} p_{12}^{2}(-z)
$$

Note that the highest-degree terms of $z^{-1} p_{12}^{2}(z)$ and $z^{-1} p_{12}^{2}(-z)$ are equal and of odd degree and so $z^{-1} p_{12}^{2}(z)-z^{-1} p_{12}^{2}(-z)$ has degree at most $2 \operatorname{deg}\left(p_{12}(z)\right)-2$. Since $\theta_{k k}=$ 1 because of the orthogonality, $2 \operatorname{deg}\left(p_{12}\right)-2 \geq \operatorname{deg}\left(p_{11}^{2}\right)=2 \operatorname{deg}\left(p_{11}\right)$. Thus $\operatorname{deg}\left(p_{12}\right)-$ $1 \geq \operatorname{deg}\left(p_{11}\right)$. Using Lemma 2.6, we see that

$$
\operatorname{degL}\left(p_{12}\right)=2 \operatorname{deg}\left(p_{12}\right)-1 \geq 2\left(\operatorname{deg}\left(p_{11}\right)+1\right)-1=\operatorname{degL}\left(p_{11}\right)+1
$$

Proving the second claim is very similar. Using (1.10),

$$
\theta_{22}=z^{2} p_{21}^{2}(z)+z^{2} p_{21}^{2}(-z)+z p_{22}^{2}(z)-z p_{22}^{2}(-z)
$$


and noting that $z p_{22}^{2}(z)-z p_{22}^{2}(-z)$ has degree at most $2 \operatorname{deg}\left(p_{22}\right)$, we see that $2 \operatorname{deg}\left(p_{22}\right) \geq 2 \operatorname{deg}\left(p_{21}\right)+2$. Using Lemma 2.6, we see that

$$
\operatorname{degL}\left(p_{22}\right)=2 \operatorname{deg}\left(p_{22}\right)+1 \geq 2 \operatorname{deg}\left(p_{21}\right)+3=\operatorname{degL}\left(p_{21}\right)+1
$$

The following result says that we can shift a function $\phi$ with vanishing moments without affecting the vanishing moments, provided that $\int_{\mathbb{R}} \phi=0$. This will simplify some of our proofs.

Proposition 2.9. If $\int_{\mathbb{R}} x^{k} f(x) d x=0$ for $k=0,1, \ldots, L-1$, then $\int_{\mathbb{R}} x^{k} f(x-S) d x=0$ for any $S$.

Proof. Substituting $u=x-S$, apply the binomial theorem and hypothesis: $\int_{\mathbb{R}} x^{k} f(x-S) d x=\int_{\mathbb{R}}(u+S)^{k} f(u) d u=\sum_{j=0}^{k}\left(\begin{array}{l}k \\ j\end{array}\right) S^{k-j} \int_{\mathbb{R}} u^{j} f(u) d u=0$.

3. Support bounds for orthogonal, symmetric scaling vectors with vanishing moments. We now limit discussion to scaling vectors with $r=2$ component functions.

If orthogonal $\Phi=\left(\phi_{1}, \phi_{2}\right)^{T}$ has some kind of symmetry and a given number of vanishing moments, we can characterize the type of symmetry for $\Phi$, give some information about the form of the symbol $P(z)$, and place some bounds on the support of each $\phi_{i}$.

We also demonstrate how to construct an $L^{2}(\mathbb{R})$ orthogonal symmetric scaling vector with minimal support for one vanishing moment. This support length depends on the type of symmetry.

We first introduce some standard notation about support for scaling vectors needed for our analysis of the interplay between vanishing moments and support. We will say that the support of $\Phi=\left(\phi_{1}, \ldots, \phi_{r}\right)^{T}$ is $\operatorname{supp}(\Phi)=\cup_{i} \operatorname{supp}\left(\phi_{i}\right)$, with each $\operatorname{supp}\left(\phi_{i}\right)$ being the convex hull of the support points of $\phi_{i}$, as in [9]. The support length of $\phi_{i}$, denoted by $\operatorname{suppL}\left(\phi_{i}\right)$, is the length of the interval $\operatorname{supp}\left(\phi_{i}\right)$.

A family $\left\{f_{i}\right\}$ of functions on $\mathbb{R}$ is locally linearly independent (LLI) if $\sum_{i} c_{i} f_{i}(x)=0$ on any nontrivial interval $I$ implies that $c_{i}=0$ for all $i$ for which $\operatorname{supp}\left(f_{i}\right) \cap I \neq \varnothing$. We say a scaling vector $\Phi$ is LLI if the family $\left\{\phi_{i}(x-k): 1 \leq i \leq r, k \in \mathbb{Z}\right\}$ is LLI. Wavelets and the LLI property have been studied together [4, 9], and So and Wang [9] proved the following result about the connection between the symbol $P(z)$ entries' degrees and each $\operatorname{supp}\left(\phi_{i}\right)$. This result allows us to put some bounds on the support length of $\phi_{i}$.

Theorem 3.1. Suppose $\Phi=\left(\phi_{1}, \phi_{2}\right)^{T}$ is LLI. Then $\operatorname{supp}\left(\phi_{i}\right)=\left[L_{i}, R_{i}\right]$ for $i=1,2$, where

$$
\begin{aligned}
& R_{1}=\max \left\{H_{11}, \frac{2 H_{12}+H_{21}}{3}, \frac{H_{12}+H_{22}}{2}\right\}, \quad L_{1}=\min \left\{B_{11}, \frac{2 B_{12}+B_{21}}{3}, \frac{B_{12}+B_{22}}{2}\right\}, \\
& R_{2}=\max \left\{H_{22}, \frac{2 H_{21}+H_{12}}{3}, \frac{H_{11}+H_{21}}{2}\right\}, \quad L_{2}=\min \left\{B_{22}, \frac{2 B_{21}+B_{12}}{3}, \frac{B_{21}+B_{11}}{2}\right\},
\end{aligned}
$$

$H_{i j}$ and $B_{i j}$ being the highest and lowest degrees, respectively, of $p_{i j}(z)$. 
COROLlary 3.2. Suppose $\Phi=\left(\phi_{1}, \phi_{2}\right)^{T}$ is LLI. Then

$$
\begin{aligned}
& \operatorname{suppL}\left(\phi_{1}\right) \geq \max \left\{\operatorname{degL}\left(p_{11}\right), \frac{2 \operatorname{degL}\left(p_{12}\right)+\operatorname{degL}\left(p_{21}\right)}{3}, \frac{\operatorname{degL}\left(p_{12}\right)+\operatorname{degL}\left(p_{22}\right)}{2}\right\}, \\
& \operatorname{suppL}\left(\phi_{2}\right) \geq \max \left\{\operatorname{degL}\left(p_{22}\right), \frac{2 \operatorname{degL}\left(p_{21}\right)+\operatorname{degL}\left(p_{12}\right)}{3}, \frac{\operatorname{degL}\left(p_{11}\right)+\operatorname{degL}\left(p_{21}\right)}{2}\right\} .
\end{aligned}
$$

Proof. Since $\operatorname{degL}\left(p_{i j}\right)=H_{i j}-B_{i j}$, Theorem 3.1 yields $\operatorname{suppL}\left(\phi_{1}\right)=R_{1}-L_{1} \geq$ $(1 / 3)\left(2 H_{12}+H_{21}\right)-(1 / 3)\left(2 B_{12}+B_{21}\right)=(2 / 3) \operatorname{degL}\left(p_{12}\right)+(1 / 3) \operatorname{degL}\left(p_{21}\right)$. This proves that $\operatorname{suppL}\left(\phi_{1}\right) \geq \operatorname{degL}\left(p_{11}\right),(1 / 2)\left(\operatorname{degL}\left(p_{12}\right)+\operatorname{degL}\left(p_{22}\right)\right)$ are very similar, which gives us the first claim. The proof of the second claim is the same.

The next two lemmas contain useful information about scaling vectors with vanishing moments.

LEMMA 3.3. Suppose symmetric, orthogonal scaling vector $\Phi=\left(\phi_{1}, \phi_{2}\right)^{T}$ has accuracy $p \geq 1$ and at least one vanishing moment. If $\phi_{1}$ is symmetric about 0 , then $\hat{\Phi}(1)$ must be a multiple of $(1,0)^{T}, P(1)$ has a one-dimensional right 1-eigenspace, and $\mathbf{y}^{0}$ of Theorem 1.2 must be a nonzero scalar multiple of $\hat{\Phi}(1)$.

Proof. By Proposition 2.3, Corollary 2.4, and Theorem 2.5, $\phi_{2}$ must be either antisymmetric or symmetric about some $\beta \neq 0$. In either case, $\hat{\phi}_{2}(1)=\int_{\mathbb{R}} \phi_{2}=0$, so $\hat{\Phi}(1)$ must be multiple of $(1,0)^{T}$ and $P(1) \hat{\Phi}(1)=\hat{\Phi}(1)$; so

$$
P(1)=\left(\begin{array}{ll}
1 & x \\
0 & y
\end{array}\right) .
$$

By Definition 1.1, the eigenvalue $y$ must be 1 or less than 1 in magnitude. For the sake of contradiction, suppose that $y=1$. Then $P(1)$ has two linearly independent eigenvectors $\hat{F}^{1}(1), \hat{F}^{2}(1)$ corresponding to two linearly independent scaling vector solutions $F^{1}, F^{2}$ by [5, Theorem 2.3]. Thus one of these, say $F^{2}=\left(f_{1}^{2}, f_{2}^{2}\right)^{T}$, must satisfy $\hat{F}^{2}(1)=(a, b)^{T}$ for some $b \neq 0$. But then its second component function $\hat{f}_{2}^{2}(1)=\int_{\mathbb{R}} f_{2}^{2}=b \neq 0$. On the other hand, since $\Phi$ is symmetric and orthogonal, its symbol $P$ has the form requiring all other scaling vector solutions to be symmetric and orthogonal, by Theorems 1.4 and 1.3 , with the first component function symmetric about 0 . So, just as with $\Phi, f_{2}^{2}$ must be either antisymmetric, or symmetric about some $\beta \neq 0$, and so $\hat{f}_{2}^{2}(1)=\int_{\mathbb{R}} f_{2}^{2}=0$. This is a contradiction; so $|y|<1$, and $P(1)$ has a one-dimensional right 1-eigenspace.

To see the final claim, multiply the orthogonality condition (1.8), evaluated at $z=1$, on the left by $\left(\mathbf{y}^{0}\right)^{T}$ from Theorem 1.2. Recalling that $\left(\mathbf{y}^{0}\right)^{T} P(1)=\left(\mathbf{y}^{0}\right)^{T}$ and $\left(\mathbf{y}^{0}\right)^{T} P(-1)$ $=\mathbf{0}$, this reduces to $\left(\mathbf{y}^{0}\right)^{T} P^{*}(1)+\mathbf{0} \cdot P^{*}(-1)=\left(\mathbf{y}^{0}\right)^{T}$. Taking transposes gives $P(1) \mathbf{y}^{0}=$ $\mathbf{y}^{0}$; so $\mathbf{y}^{0}$; is a right 1-eigenvector of $P(1)$. Thus $\mathbf{y}^{0}$ must be a multiple of $\hat{\Phi}(1)$.

LEMMA 3.4. Suppose $\Phi=\left(\phi_{1}, \phi_{2}\right)^{T}$ has $L-1 \geq 1$ vanishing moments and $\hat{\Phi}(1)=$ $A(1,0)^{T}$ for some nonzero constant $A$. Then the entries $p_{11}$ and $p_{21}$ of symbol $P(z)$ 
satisfy

$$
\begin{aligned}
& p_{11}(z)=1+(1-z)^{L} Q_{11}(z), \\
& p_{21}(z)=(1-z)^{L} Q_{21}(z)
\end{aligned}
$$

for polynomials $Q_{11}, Q_{21}$.

Proof. Since $P(1) \hat{\Phi}(1)=\hat{\Phi}(1)$, we must have $p_{11}(1)=1$ and $p_{21}(z)=0$. By Theorem 2.2 ,

$$
D^{k} P(1)\left(\begin{array}{l}
1 \\
0
\end{array}\right)=\left(\begin{array}{l}
0 \\
0
\end{array}\right)
$$

for $k=1, \ldots, L-1$. Thus $(1-z)^{L}$ must divide $p_{11}(z)-1$ and $p_{21}(z)$.

The first symmetric, orthogonal, continuous, compactly supported scaling vector $\Phi=\left(\phi_{1}, \phi_{2}\right)^{T}$ in the literature, constructed in [3], had $\phi_{1}$ symmetric about an integer and $\phi_{2}$ symmetric about $1 / 2$. The next result gives necessary conditions on a scaling vector's symmetry, symbol, and support length if we insist additionally on vanishing moments. In particular, neither $\phi_{1}$ nor $\phi_{2}$ can be antisymmetric.

THEOREM 3.5. Suppose symmetric, orthogonal, LLI scaling vector $\Phi=\left(\phi_{1}, \phi_{2}\right)^{T}$ has accuracy $p \geq 1$ and $L-1 \geq 1$ vanishing moments. If $\phi_{2}$ is symmetric or antisymmetric about $m+1 / 2$ for integer $m$, then

(1) $\phi_{1}$ is symmetric about 0 ;

(2) $\phi_{2}$ is symmetric;

(3) if $L$ is even, then $\operatorname{suppL}\left(\phi_{1}\right) \geq L+1, \operatorname{suppL}\left(\phi_{2}\right) \geq L+1$, and $P(z)$ has the form

$$
P(z)=\left(\begin{array}{cc}
1+(1-z)^{L} Q_{11}(z) & \left(1-z^{2}\right)(1-z) R_{12}(z) \\
(1-z)^{L} Q_{21}(z) & (1+z) R_{22}(z)
\end{array}\right)
$$

(4) if $L$ is odd, then $\operatorname{suppL}\left(\phi_{1}\right) \geq L+2, \operatorname{suppL}\left(\phi_{2}\right) \geq L+2$, and $P(z)$ has the form

$$
P(z)=\left(\begin{array}{cc}
1+(1-z)^{L+1} R_{11}(z) & \left(1-z^{2}\right)(1-z) R_{12}(z) \\
(1-z)^{L+1} R_{21}(z) & (1+z) R_{22}(z)
\end{array}\right)
$$

Proof. By Corollary 2.4, $\phi_{1}$ must be symmetric about 0 , thus proving claim (1).

From Lemma 3.3, $\int_{\mathbb{R}} \phi_{2}=0$, so by Propositions 1.6 and 2.9, we may assume WLOG that this integer $m$ is zero.

Suppose $\phi_{2}$ is antisymmetric for the sake of contradiction. From (1.10) with $T_{2}=1 / 2$, $p_{21}(z)=-z^{2} p_{21}(1 / z)$ and $p_{22}(z)=z p_{22}\left(z^{-1}\right)$. Evaluating at $z= \pm 1$, we see $p_{21}( \pm 1)=0$ and $p_{22}(-1)=0$. Evaluating the $2-2$ position of (1.8) at $z=1$ simplifies to $\left(p_{22}(1)\right)^{2}=1$. But $P(1)=\left(\begin{array}{cc}1 & x \\ 0 & p_{22}(1)\end{array}\right)$, so Definition 1.1 says $p_{22}(1)=1$. This says $P(0)$ has a two-dimensional right 1-eigenspace, which is a contradiction of Lemma 3.3. This says that $\phi_{2}$ is in fact symmetric, thus completing the proof of 2 .

To prove claims (3) and (4), first note that by Lemma 3.3 and Theorem $1.2,\left(\mathbf{y}^{0}\right)^{T} P(1)=$ $\left(\mathbf{y}^{0}\right)^{T}=A(1,0)^{T}=A\left(p_{11}(1), p_{12}(1)\right)$; so we must have $1-z$ as a factor of $p_{12}(z)$. 
Similarly $\left(\mathbf{y}^{0}\right)^{T} P(-1)=A\left(p_{11}(-1), p_{12}(-1)\right)=\mathbf{0}$ says that we must have $1+z$ as a factor of $p_{12}(z)$.

By Lemma 3.4 and (1.10) with $T_{2}=1 / 2$, we see

$$
\begin{aligned}
& (1-z)^{L} Q_{11}(z)=\left(1-z^{-1}\right)^{L} Q_{11}\left(z^{-1}\right), \quad z p_{12}(z)=p_{12}\left(z^{-1}\right), \\
& (1-z)^{L} Q_{21}(z)=z^{2}\left(1-z^{-1}\right)^{L} Q_{21}\left(z^{-1}\right), \quad p_{22}(z)=z p_{22}\left(z^{-1}\right) .
\end{aligned}
$$

Multiplying the 1-1 and 2-1 positions by $z^{L}$ and letting $p_{12}(z)=\left(1-z^{2}\right) Q_{12}$, we see that

$$
\begin{array}{ll}
z^{L} Q_{11}(z)=(-1)^{L} Q_{11}\left(z^{-1}\right), & z^{3} Q_{12}(z)=-Q_{12}\left(z^{-1}\right), \\
z^{L} Q_{21}(z)=(-1)^{L} z^{2} Q_{21}\left(z^{-1}\right), & p_{22}(-1)=0
\end{array}
$$

so $1+z$ divides $p_{22}(z)$ and $Q_{12}(1)=0$; so $Q_{12}(z)=(1-z){ }_{12} R(z)$ for some $R_{12}(z)$.

Consider the case when $L$ is even. The form of $P(z)$ has been verified. Using Proposition 2.8, $\operatorname{degL}\left(p_{12}\right) \geq \operatorname{degL}\left(p_{11}\right)+1 \geq L+1$ and $\operatorname{degL}\left(p_{22}\right) \geq \operatorname{degL}\left(p_{21}\right)+1 \geq$ $L+1$; so by Corollary 3.2, $\operatorname{suppL}\left(\phi_{2}\right), \operatorname{suppL}\left(\phi_{1}\right) \geq L+1$.

Consider the case when $L$ is odd. In this case, we see from (3.9) that $Q_{11}(1)=$ $Q_{21}(1)=0$; so $1-z$ divides both $Q_{11}(z)$ and $Q_{21}(z)$. This proves the form of $P(z)$ and $\operatorname{degL}\left(p_{21}\right), \operatorname{degL}\left(p_{11}\right) \geq L+1$. Using Proposition $2.8, \operatorname{degL}\left(p_{22}\right) \geq \operatorname{degL}\left(p_{21}\right)+1 \geq L+2$ and $\operatorname{degL}\left(p_{12}\right) \geq \operatorname{degL}\left(p_{11}\right)+1 \geq L+2$; so by Corollary $3.2, \operatorname{suppL}\left(\phi_{2}\right) \geq L+2$ and $\operatorname{suppL}\left(\phi_{1}\right) \geq(1 / 2) \operatorname{degL}\left(p_{12}\right)+(1 / 2) \operatorname{degL}\left(p_{22}\right) \geq L+2$.

EXAMPLE 3.6. Set $L=2$. We use Theorem 3.5 to guide the construction of an $L^{2}(\mathbb{R})$ orthogonal symmetric scaling vector with minimal support for one vanishing moment. Naturally $\phi_{1}$ is symmetric about 0 and $\phi_{2}$ is symmetric about $1 / 2$. We begin with $Q_{21}(z)$ being a monomial. By Proposition $2.8, \operatorname{degL}\left(p_{22}\right) \geq \operatorname{degL}\left(p_{21}\right)+1=3$, so we try $\operatorname{degL}\left(R_{22}\right)=2$. Brute force with (1.8) and Lemma 2.6 shows that orthogonality cannot be obtained if $\operatorname{degL}\left(Q_{11}\right)<2$; so we try $\operatorname{degL}\left(Q_{11}\right)=2$ and thus $\operatorname{degL}\left(R_{12}\right)=2$ is minimal. We put the unknowns $Q_{11}, Q_{21}, R_{12}, R_{22}$ into form ensuring the symmetry conditions, and solved for the parameters to guarantee orthogonality, with the help of Mathematica:

$$
\begin{aligned}
& P(z)=\left[\begin{array}{cc}
1+\frac{(1-z)^{2}}{z^{2}}\left(a_{11}+b_{11} z+a_{11} z^{2}\right) & \frac{\left(1-z^{2}\right)}{z^{3}}(1-z)\left(a_{12}+b_{12} z+a_{12} z^{2}\right) \\
a_{21}(1-z)^{2} & \frac{(1+z)}{z}\left(a_{22}+b_{22} z+a_{22} z^{2}\right)
\end{array}\right], \\
& a_{11} \approx-0.05176, b_{11} \approx 0.14648, a_{12} \approx-0.03832, b_{12} \approx-0.00337 \\
& a_{21} \approx-0.24986, a_{22} \approx-0.185, b_{22} \approx 0.16874 .
\end{aligned}
$$

Theorem 1.2 was used to verify that the solution was indeed $L^{2}(\mathbb{R})$. The supports $\operatorname{suppL}\left(\phi_{1}\right)=4, \operatorname{suppL}\left(\phi_{2}\right)=3$ are minimal for the conditions put on $\Phi$. The plots of $\phi_{1}$ and $\phi_{2}$ are given in Figure 3.1.

We next consider necessary conditions for an orthogonal scaling vector with vanishing moments if both $\phi_{1}$ and $\phi_{2}$ have symmetry centered about integers. In particular, 

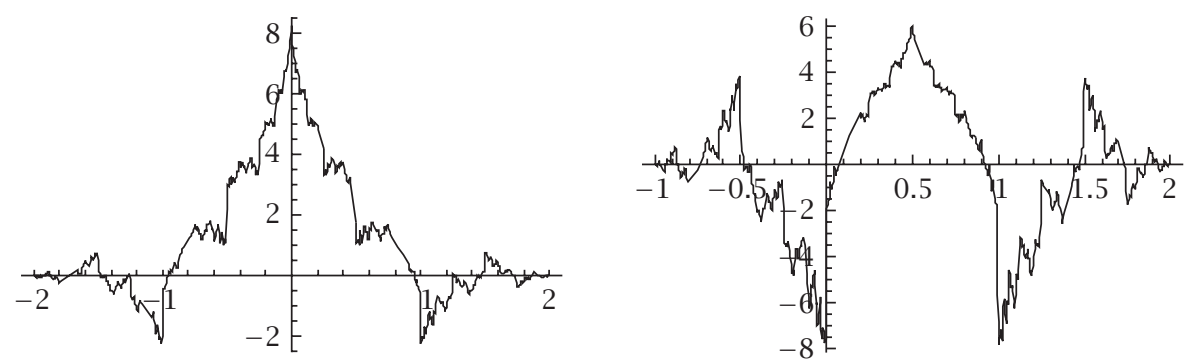

FIGURE 3.1. Scaling functions for Example 3.6.

we find that one scaling function must be symmetric about zero and the other must be antisymmetric.

THEOREM 3.7. Suppose symmetric, orthogonal, LLI scaling vector $\Phi=\left(\phi_{1}, \phi_{2}\right)^{T}$ has accuracy $p \geq 1$ and $L-1 \geq 1$ vanishing moments. If $\phi_{1}$ is symmetric about an integer and $\phi_{2}$ is (anti)symmetric about integer, then

(1) $\phi_{2}$ must be antisymmetric;

(2) $\phi_{1}$ is symmetric about zero;

(3) if $L$ is even, then $\operatorname{suppL}\left(\phi_{1}\right) \geq L+1, \operatorname{suppL}\left(\phi_{2}\right) \geq L+2$, and the symbol $P$ has the form

$$
P(z)=\left(\begin{array}{cc}
1+(1-z)^{L} Q_{11}(z) & \left(1-z^{2}\right) Q_{12}(z) \\
(1-z)^{L}\left(1-z^{2}\right) R_{21}(z) & P_{22}(z)
\end{array}\right)
$$

(4) if $L$ is odd, then $\operatorname{suppL}\left(\phi_{1}\right) \geq L+1, \operatorname{suppL}\left(\phi_{2}\right) \geq L+1$, and $P(z)$ has the form

$$
P(z)=\left(\begin{array}{cc}
1+(1-z)^{L+1} Q_{11}(z) & \left(1-z^{2}\right) Q_{12}(z) \\
(1-z)^{L}(1+z) R_{21}(z) & P_{22}(z)
\end{array}\right)
$$

Proof. By Theorem 2.5, $\phi_{2}$ must be antisymmetric about an integer. Since $\int_{\mathbb{R}} \phi_{2}=0$, by Proposition 1.6, we may assume without loss of generality that this integer is zero. Claim (2) follows from Corollary 2.4.

By Lemma 3.4 and (1.10), we see that

$$
\begin{array}{lc}
(1-z)^{L} Q_{11}(z)=\left(1-z^{-1}\right)^{L} Q_{11}\left(z^{-1}\right), & p_{12}(z)=-p_{12}\left(z^{-1}\right), \\
(1-z)^{L} Q_{21}(z)=-\left(1-z^{-1}\right)^{L} Q_{21}\left(z^{-1}\right), & p_{22}(z)=p_{22}\left(z^{-1}\right) .
\end{array}
$$

It follows that

$$
\begin{gathered}
z^{L} Q_{11}(z)=(-1)^{L} Q_{11}\left(z^{-1}\right), \\
z^{L} Q_{21}(z)=(-1)^{L+1} Q_{21}\left(z^{-1}\right), \\
p_{12}( \pm 1)=0 \Longrightarrow 1-z^{2} \text { divides } P_{12}(z) .
\end{gathered}
$$


Consider the case where $L$ is even. Then $Q_{21}( \pm 1)=0$, so $1-z^{2}$ divides $Q_{21}$. Thus $(1-z)^{L}\left(1-z^{2}\right)$ divides $P_{21}(z)$, so $\operatorname{degL}\left(P_{21}\right) \geq L+2$. From Proposition 2.7 , we see $\operatorname{degL}\left(P_{22}\right)$ is also at least $L+2$. Thus $\operatorname{suppL}\left(\phi_{2}\right) \geq L+2$.

Now $\operatorname{degL}\left(P_{11}\right)$ is clearly at least $L$, so $\operatorname{degL}\left(P_{12}\right) \geq L$. Then Corollary 3.2 says $\operatorname{suppL}\left(\phi_{1}\right) \geq(1 / 2) \operatorname{degL}\left(P_{12}\right)+(1 / 2) \operatorname{degL}\left(P_{22}\right) \geq L+1$.

Consider the case where $L$ is odd. Then $Q_{11}(1)=0$, so $1-z$ divides $Q_{11}$. Thus $(1-z)^{L+1}$ divides $P_{11}(z)-1$, so $\operatorname{degL}\left(P_{11}\right) \geq L+1$ and thus $\operatorname{suppL}\left(\phi_{1}\right) \geq L+1$. Next, note that $Q_{21}(-1)=0$, so $(1-z)^{L}(1+z)$ divides $P_{21}$ and thus $\operatorname{deg} L\left(P_{21}\right) \geq L+1$. Now by Corollary 3.2, $\operatorname{suppL}\left(\phi_{2}\right) \geq(1 / 2) \operatorname{degL}\left(P_{11}\right)+(1 / 2) \operatorname{degL}\left(P_{21}\right) \geq L+1$.

EXAMPLE 3.8. Set $L=2$. We use this theorem to guide a construction of an $L^{2}(\mathbb{R})$ LLI, orthogonal, symmetric scaling vector with one vanishing moment and minimal support. Naturally $\phi_{1}$ is symmetric about 0 and $\phi_{2}$ is antisymmetric about $1 / 2, \operatorname{suppL}\left(\phi_{1}\right) \geq 3$, $\operatorname{suppL}\left(\phi_{2}\right) \geq 4$. We begin with symbol of the form given in Theorem 3.7 part 3. Brute force with (1.8) shows that orthogonality cannot be obtained if $\operatorname{degL}\left(Q_{11}\right)<2$, so we try $\operatorname{degL}\left(Q_{11}\right)=2$ and thus $\operatorname{degL}\left(Q_{12}\right)=2$ is minimal. We put the unknowns $Q_{11}, Q_{12}$, $P_{22}, R_{21}$ into form ensuring the symmetry conditions, and solved for the parameters to guarantee orthogonality, with the help of Mathematica. Theorem 1.2 was used to verify that the solution was indeed $L^{2}(\mathbb{R})$. The supports $\operatorname{suppL}\left(\phi_{1}\right)=4, \operatorname{suppL}\left(\phi_{2}\right)=4$ are minimal for the conditions required for $\Phi$.

\section{REFERENCES}

[1] A. Cohen, I. Daubechies, and G. Plonka, Regularity of refinable function vectors, J. Fourier Anal. Appl. 3 (1997), no. 3, 295-324.

[2] I. Daubechies, Ten Lectures on Wavelets, CBMS-NSF Regional Conference Series in Applied Mathematics, vol. 61, Society for Industrial and Applied Mathematics (SIAM), Pennsylvania, 1992.

[3] J. S. Geronimo, D. P. Hardin, and P. R. Massopust, Fractal functions and wavelet expansions based on several scaling functions, J. Approx. Theory 78 (1994), no. 3, 373-401.

[4] T. N. T. Goodman, R.-Q. Jia, and D.-X. Zhou, Local linear independence of refinable vectors of functions, Proc. Roy. Soc. Edinburgh Sect. A 130 (2000), no. 4, 813-826.

[5] C. Heil and D. Colella, Matrix refinement equations: existence and uniqueness, J. Fourier Anal. Appl. 2 (1996), no. 4, 363-377.

[6] P. R. Massopust, D. K. Ruch, and P. J. Van Fleet, On the support properties of scaling vectors, Appl. Comput. Harmon. Anal. 3 (1996), no. 3, 229-238.

[7] G. Plonka and V. Strela, Construction of multiscaling functions with approximation and symmetry, SIAM J. Math. Anal. 29 (1998), no. 2, 481-510.

[8] D. K. Ruch, Constructing multicoiflets, Joint MAA-AMS Meetings, Texas, 1999.

[9] W. So and J. Wang, Estimating the support of a scaling vector, SIAM J. Matrix Anal. Appl. 18 (1997), no. 1, 66-73.

[10] V. Strela, Multiwavelets: regularity, orthogonality, and symmetry via two-scale similarity transform, Stud. Appl. Math. 98 (1997), no. 4, 335-354.

David K. Ruch: Department of Mathematical and Computer Sciences, Metropolitan State College of Denver, Denver, CO 80217, USA

E-mail address: ruch@mscd. edu 


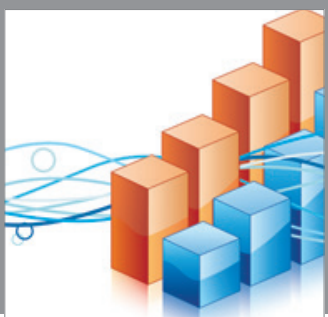

Advances in

Operations Research

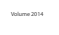

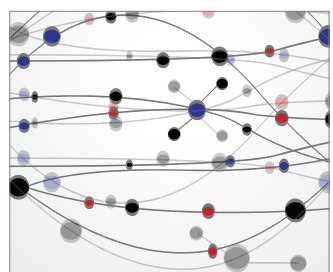

\section{The Scientific} World Journal
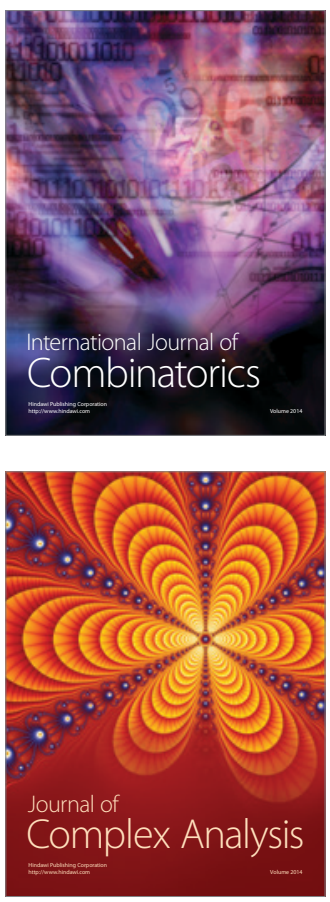

International Journal of

Mathematics and

Mathematical

Sciences
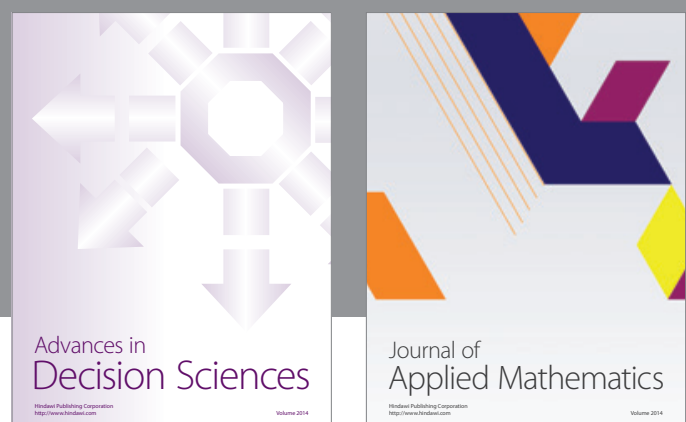

Journal of

Applied Mathematics
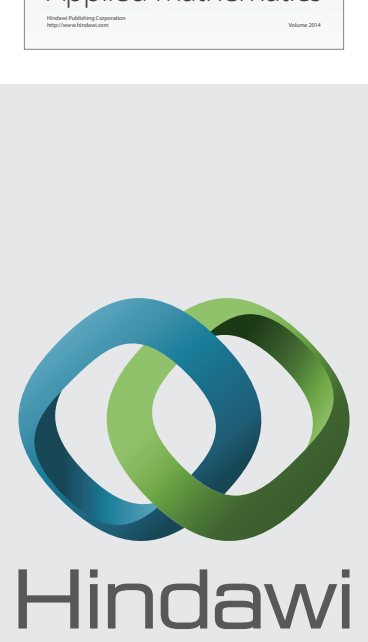

Submit your manuscripts at http://www.hindawi.com
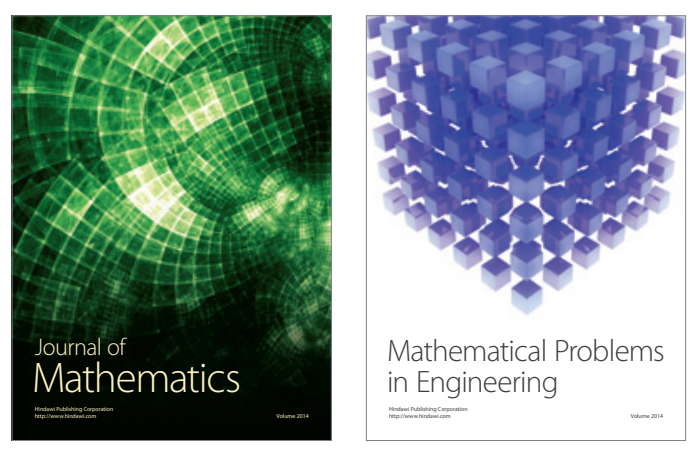

Mathematical Problems in Engineering
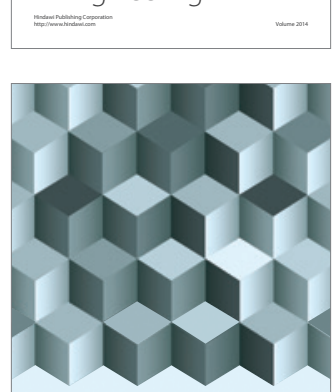

Journal of

Function Spaces
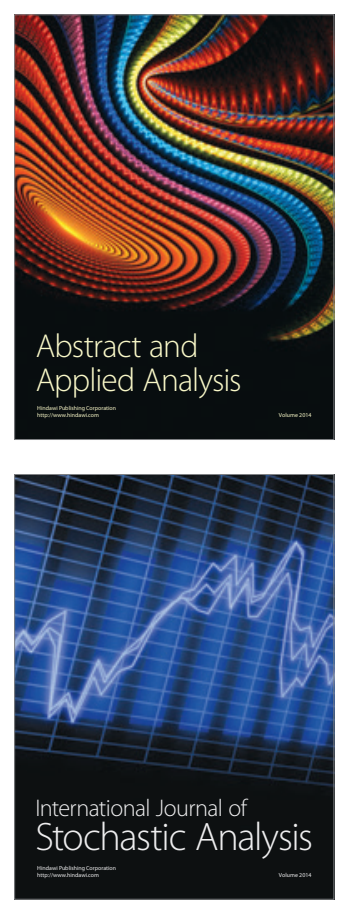

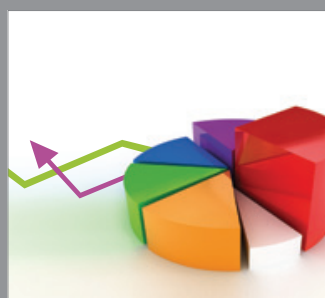

ournal of

Probability and Statistics

Promensencen
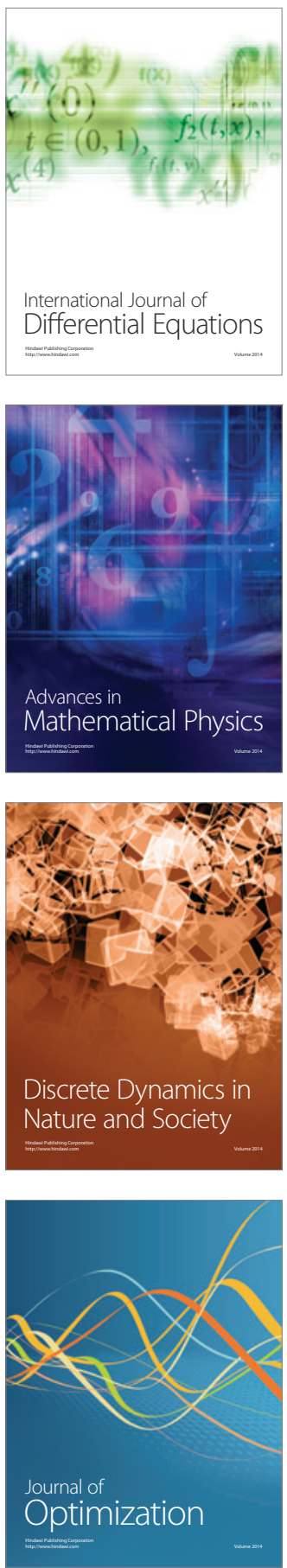